\title{
Non-Destructive Material Homogeneity Evaluation Using Scanning Millimeter Wave Microscopy
}

\author{
A. LAurinaviČIUS ${ }^{a, *}$, T. AnBinderis ${ }^{b}$, J. IKONIKOVA $^{b}$ \\ AND YU. PRISHUTOV ${ }^{b}$ \\ ${ }^{a}$ Semiconductor Physics Institute \\ A. Goštauto 11, 01108 Vilnius, Lithuania \\ ${ }^{b}$ JSC "ELMIKA", Naugarduko 41, 03227 Vilnius, Lithuania
}

Millimeter wave bridge technique for non-destructive material homogeneity characterization is described. The idea of this technique is the local excitation of the millimeter waves in the testing material and the measurement of the transmitted (reflected) wave amplitude and phase in different places of it, i.e. the material plate is scanned by the beam of the millimeter waves. The results of the homogeneity measurements for dielectric wafers according to dielectric constant anisotropy are presented. The measurement technique sensitivity is discussed.

PACS numbers: 78.70.Gq, 81.70.Ex

\section{Introduction}

In many cases, the quality of the fabricated material depends on spatial distribution of electrical, dielectric or mechanical parameters in the whole area of the sample. This is especially important for large area dielectric substrates and thin films used in electronics. Relatively short wavelength of the millimeter wave provides the possibility to utilize them for non-destructive homogeneity characterization of materials $[1,2]$. In the present paper the new possibilities of the technique based on the scanning of the material by millimeter wave beam and the measurement of transmitted (reflected) amplitude and phase in different places are discussed.

It is known that electromagnetic wave transmitted through a dielectric plate demonstrates resonance character. Maximum of transmission is observed at the condition $d / \lambda=1 / 2,1,3 / 2,2, \ldots n / 2$, where $\mathrm{d}$ is a thickness of the plate, $\lambda$ is a

*corresponding author; e-mail: laurinavicius@pfi.lt 
wavelength, $n$ is an integer. This resonance is known as the Fabry-Perot resonance. An analysis of electromagnetic wave transmission through the dielectric plate was presented recently [3].

\section{Measurement technique}

The main idea of the measurement technique operation is the local excitation of millimeter waves in the sample under test and the measurement of transmitted (reflected) wave amplitude and phase at different points of the sample. In essence, we use a millimeter wave bridge consisting of a reference signal and measuring signal channels (Fig. 1). The tested sample is placed between waveguide probes

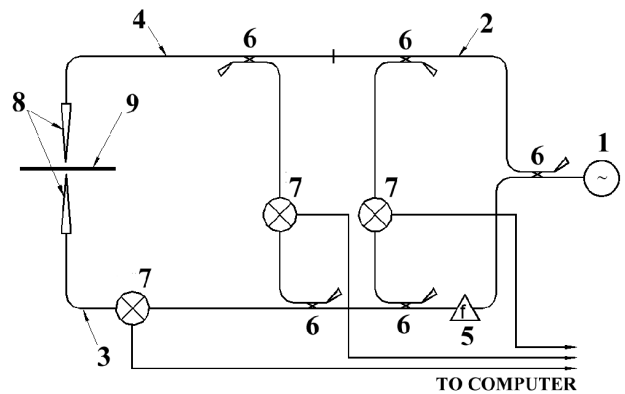

Fig. 1. Schematic diagram of the device measuring the transmitted and reflected electromagnetic wave amplitude and phase: 1 is millimeter wave oscillator, 2 is reference signal channel, 3 is transmitted signal channel, 4 is reflected signal channel, 5 is frequency converter, 6 are directional couplers, 7 are mixers, 8 are antennas, and 9 is the sample under the test.

that provide both local excitation and reception of the low power millimeter wave signals. The sample can be moved relative to the exciting and receiving probes by scanning mechanism. Changes of the electric, dielectric or mechanical parameters in the sample area cause changes in the amplitude and phase of the transmitted (reflected) signal. By probing the sample at different points with the millimeter wave beam, information about the homogeneity of the sample can be obtained. All measurement processes are computer controlled and the measurement results are compiled in the computer. Some examples of the applications of our technique for the measurement of dielectric constant anisotropy distribution in the wafers are presented below.

\section{Measurement results}

$\mathrm{LiNbO}_{3}$ and sapphire wafers have been used for dielectric constant anisotropy measurement tests. Measurements were performed in the frequency range 120 $150 \mathrm{GHz}$. Open end sections of rectangular waveguide were used for local excitation and reception of millimeter waves. Scanning of the sample was performed 


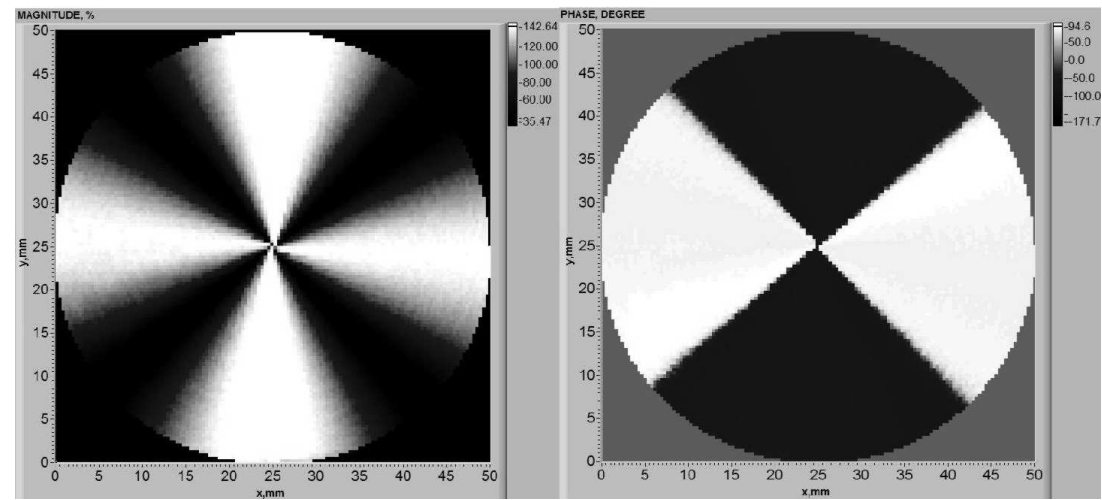

Fig. 2. Transmitted millimeter wave amplitude (left) and phase (right) images of the $\mathrm{LiNbO}_{3}$ wafer at frequency $130 \mathrm{GHz}$. Thickness of the wafer is $1.00 \mathrm{~mm}$. Diameter of the scanning area is $50 \mathrm{~mm}$.
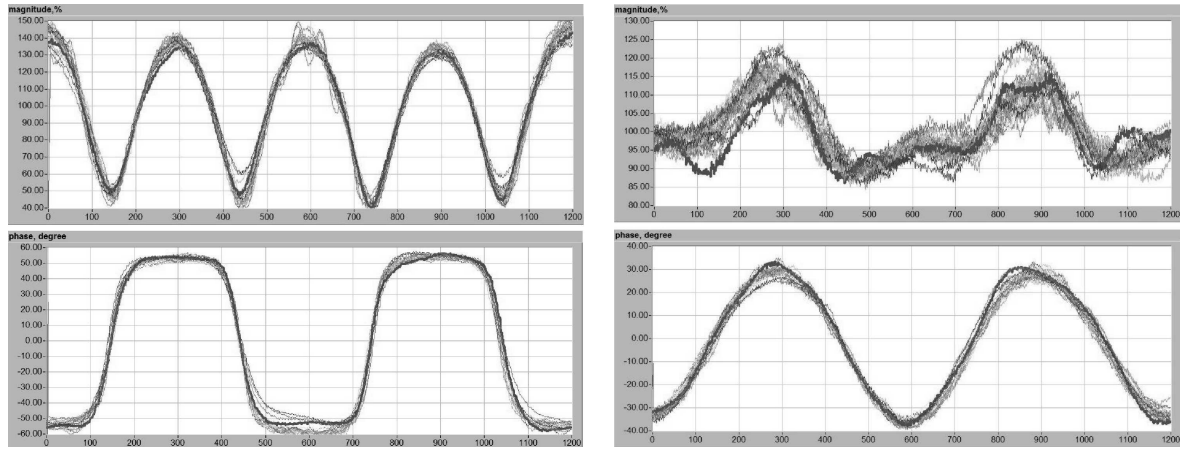

Fig. 3. Transmitted wave amplitude (top) and phase (bottom) dependences for the sapphire wafer on a number of measurement points on the rotation helix at frequency $122 \mathrm{GHz}$ (left part) and $132 \mathrm{GHz}$ (right part). Thickness of the wafer is $1.85 \mathrm{~mm}$.

using two step motors. One of them serves for the sample rotation and the other one provides its linear motion. Therefore, the scanning process is going on the helix way (Fig. 1) covering the whole surface of the sample. Significant variation in the amplitude and phase of the transmitted millimeter wave has been observed in the corresponding images for $\mathrm{LiNbO}_{3}$ wafer (Fig. 2). They are related with dielectric constant anisotropy of the crystal when its orientation changes according to exciting wave electric vector polarization. Measurement results of the sapphire wafer for two different frequencies are shown in Fig. 3. Such amplitude and phase variation data are used for constructing images like are shown in Fig. 2. Comparing curves shown on left and right parts that were measured at $f=122 \mathrm{GHz}$ and $f=132 \mathrm{GHz}$, respectively, one can see that the changes in phase are larger at frequency $122 \mathrm{GHz}\left(\Delta \varphi \approx 110^{\circ}\right)$ than at frequency $132 \mathrm{GHz}\left(\Delta \varphi \approx 66^{\circ}\right)$ of the transmitted wave. The point here is that for the tested wafer the fifth-order 
Fabry-Perot resonance condition $(d=5 \lambda / 2)$ is fulfilled at $f=122 \mathrm{GHz}$. As for $f=132 \mathrm{GHz}(d=11 \lambda / 4)$, this frequency is far away from the resonance condition. These results confirm, in general, our earlier predictions that the largest sensitivity of homogeneity mapping can be achieved when the measurements are performed at the Fabry-Perot resonance conditions [3].

\section{Conclusions}

Millimeter wave bridge technique for non-destructive material dielectric constant anisotropy characterization is presented. The idea of this technique is the local excitation of the millimeter waves in the wafer under test and the measurement of the transmitted wave amplitude and phase in the different places of the sample. Measurement results of the anisotropy measurements for some dielectric wafers are presented. The space resolution is about $1 \mathrm{~mm}^{2}$. The measurement technique sensitivity is discussed.

\section{Acknowledgments}

This work was supported by the Lithuanian Agency for International Science and Technology Development Programmes through the European Cooperation Program in the Field of Scientific and Technical Research (Action COST P17). We also thank the project MULTIMA of Lithuanian State Science and Studies Foundation (No C-18/2007).

\section{References}

[1] A. Laurinavičius, T. Anbinderis, O. Martjanova, J. Prišutov, A. Abrutis, A. Teišerskis, Int. J. Infrared Millim. Waves 22, 961 (2001).

[2] A. Laurinavičius, T. Anbinderis, Yu. Prishutov, O. Martjanova, Electron. Lett. 39, 1315 (2003).

[3] Ž. Kancleris, A. Laurinavičius, T. Anbinderis, Int. J. Infrared Millim. Waves 25, 1099 (2004). 